Research Article

\title{
Inhibition of Proinflammatory Cytokines in Cutibacterium acnes-Induced Inflammation in HaCaT Cells by Using Buddleja davidii Aqueous Extract
}

\author{
Anh Thu Nguyen and Ki-young Kim \\ Graduate School of Biotechnology, Kyung Hee University, Giheung, Yongin-si, Gyeonggi-do 1732, Republic of Korea \\ Correspondence should be addressed to Ki-young Kim; kiyoung@khu.ac.kr
}

Received 6 December 2019; Revised 3 March 2020; Accepted 31 March 2020; Published 21 April 2020

Academic Editor: Istvan Boldogh

Copyright (C) 2020 Anh Thu Nguyen and Ki-young Kim. This is an open access article distributed under the Creative Commons Attribution License, which permits unrestricted use, distribution, and reproduction in any medium, provided the original work is properly cited.

\begin{abstract}
Acne is an inflammatory skin disorder; although some anti-inflammatory medicines for treating acne are available in a market, they have considerable side effects; therefore, new treatment options are needed. In the present study, among the 16 aqueous extracts of plants collected from Jeju Island in Korea which are used to test anti-inflammatory activity, B. davidii showed the strong decline of the proinflammatory cytokine expression against the inflammatory process caused by C. acnes in Human HaCaT keratinocyte cells. B. davidii downregulated the expression of $57 \%$ of COX-2, $41 \%$ of iNOS, and proinflammatory cytokines $29 \%$ of TNF- $\alpha, 32 \%$ of IL- $1 \beta, 21 \%$ of IL- 6 , and $35 \%$ of IL- 8 . Furthermore, $B$. davidii inhibited NF- $\kappa$ B and MAPK signaling cascades in keratinocytes that activated by toll-like receptor 2 (TLR-2) in response to $C$. acnes. Given those results, $B$. davidii is a potential agent to reduce the proinflammatory cytokine expression against C. acnes-induced inflammation and might provide an alternative to the current medications.
\end{abstract}

\section{Introduction}

Acne is a chronic inflammatory disorder of the sebaceous follicles on the chin, cheek, forehead, and back. Cysts and scarring can occur when the disease is severe. Although acne is a skin disease, it can lead to psychological issues that affect patient quality of life. In acne pathogenesis, C. acnes has been identified as one of the main causative agents. C. acnes, a commensal Gram-positive anaerobic, colonizes the duct of the sebaceous follicle, causing an innate immune response [1].

Inflammation is the body's initial complex response to infection. The processes of inflammation caused by C. acnes begin when peptidoglycan (PGN) and lipoteichoic acid (LTA) recognized by binding to toll-like receptors (TLRs) activate the TLR signaling pathway and trigger important intracellular signaling pathways including the mitogen-activated protein kinase (MAPK) and nuclear factor kappa-B $(\mathrm{NF}-\kappa \mathrm{B})$ pathways. This causes nuclear translocation of transcription factors, such as activator protein-1 (AP-1) and NF- $\kappa \mathrm{B}$, ultimately leading to production of a variety of proinflammatory cytokines including tumor necrosis factor (TNF) $\alpha$, interleukin (IL) $1 \beta$, IL-8, and IL-6 $[2,3]$.

Adverse effects associated with anti-inflammatory drugs have become increasingly common and include increased risk of gastrointestinal ulcers, bleeding, heart attack, and kidney disease associated with nonsteroidal anti-inflammatory drugs (NSAIDs) [4]. Some anti-inflammatory drugs interfere with deactivated and hyperactivated cellular signaling networks or transcription factors [4]. Therefore, to limit adverse effects, the next generation of anti-inflammatory drugs needs to target either enzymes or signaling pathways that are only operational in affected tissues or a small subset of inflammatory target genes.

Medicinal plants have long been used as sources of therapeutically active compounds, which is also a popular traditional medicine to treat inflammatory diseases. The use of traditional medicines for treatment of inflammation could 
provide safer alternatives compared with existing conventional treatments. Evidence suggests that herbal medicines have a lower rate of side effects compared with conventional medicines [5]. Furthermore, medicinal plants used for dermatological purposes with low or no side effects are gaining popularity in both traditional medicine and the cosmetic industry. Buddleja davidii is a perennial herbaceous plant that belongs to the Scrophulariaceae family with a pan tropical distribution across South Asia, Africa, and America. Its roots, leaves, and flowers are used in folk medicine for antioxidant, anti-inflammation, antiviral, antiplasmodium, and antifungal effects $[6,7]$.

The purpose of the present study was to determine the anti-inflammatory activity of $B$. davidii against $C$. acnes infection and to elucidate its mechanism of action in human keratinocyte cell line.

\section{Materials and Methods}

2.1. Preparation of Bacteria. C. acnes (CCARM9009) were purchased from the Culture Collection of Antimicrobial Resistant Microbes (CCARM) at Seoul Women's University in Korea. C. acnes were grown in Reinforced Clostridial Medium (RCM) agar (Becton, Dickinson and Company, USA) for $48-72$ hours at $37^{\circ} \mathrm{C}$ under anaerobic conditions in a GasPak ${ }^{\mathrm{TM}} \mathrm{EZ}$ anaerobic container system (Becton, Dickinson and Company, USA). C. acnes were cultured in RCM broth to $100 \mathrm{MOI}$ and then heat-killed at $80^{\circ} \mathrm{C}$ for 30 minutes before use in further experiments [2].

2.2. Plant Material and Extraction. Candidate plants, the herb medicine with anti-inflammation properties, were obtained from Jeju Island in South Korea. The plants were cleaned with distilled water and then dried using a freeze dryer (iLShin, Korea). After drying, $300 \mathrm{~g}$ of the dried herb was extracted three times in 3 liter of distilled water with refluxing at $80^{\circ} \mathrm{C}$ for 8 hours. $500 \mathrm{ml}$ extract was concentrated under reduced pressure at $50^{\circ} \mathrm{C}$ using a rotary evaporator (EYELA, Japan) connected to a refrigerated bath circulator (Jeio Tech, Korea) [8]. The decoction was filtered using muslin cloth followed by Whatman (GE Healthcare, USA) grade 1 filter paper, lyophilized using a freeze dryer (iLShin, Korea), and stored at $4^{\circ} \mathrm{C}$ before further use [9]. To prepare the sample for experiments, the stock of extract powder $(10 \mathrm{mg})$ was dissolved in $1 \mathrm{ml}$ of dimethyl sulfoxide (DMSO).

2.3. Cell Culture. Human HaCaT keratinocyte cells [10] were grown in Dulbecco's Modified Eagle's Medium (DMEM) supplemented with $10 \%$ fetal bovine serum and $100 \mathrm{U} / \mathrm{ml}$ penicillin at $37^{\circ} \mathrm{C}$ in a humidified incubator under $5 \% \mathrm{CO}_{2}$.

2.4. Quantitative Real-Time PCR. HaCaT cells (seeded at a density of $5 \times 10^{5}$ per well in a six-well plate) were incubated for 24 hours, pretreated with various concentrations (12.5, 25,50 and $100 \mu \mathrm{g} / \mathrm{ml}$ ) of aqueous extract of plants for 1 hour, and subsequently treated with heat-killed C. acnes (100 MOI,
$5 \times 10^{7} \mathrm{CFU}$ per well) for 24 hours. Total RNA was isolated from the HaCaT cells using TRIzol reagent (Life Technology, Thermo Fisher Scientific, USA) according to the manufacturer's protocol. A reverse transcriptase (NanoHelix, Korea) reaction was prepared using $1 \mu \mathrm{g}$ of RNA to obtain cDNA. The cDNA was used as the template for real-time PCR (qRTPCR), which was carried out with QGreen 2X SybrGreen qPCR Master Mix (CellSafe, Korea). The primer sequences used to detect TLR-2, iNOS, COX-2, TNF- $\alpha$, IL-8, IL-6, IL$1 \beta$, and GAPDH are listed in Table 1. GAPDH was used as an endogenous control. The delta-delta $\mathrm{Cq}$ formula is used to calculate the gene expression.

2.5. Western Blot Analysis. HaCaT cells were treated with $B$. davidii aqueous extract and heat-killed $C$. acnes as described above. The cells were then lysed using radioimmunoprecipitation assay buffer (RIPA) lysis buffer containing $150 \mathrm{mM}$ sodium chloride, $1 \%$ Triton X-100, $0.5 \%$ sodium deoxycholate, $0.1 \%$ sodium dodecyl sulfate (SDS), $50 \mathrm{mM}$ Tris ( $\mathrm{pH} 8.0$ ), and complete protease inhibitor cocktail (BIOMAX, Korea). Protein concentration was determined with the Bradford ((Sigma-Aldrich, USA) assay using bovine serum albumin as a standard and detected using a UVITEC imaging system (Uvitec Ltd, UK). Protein samples $(50 \mu \mathrm{g})$ were separated by sodium dodecyl sulfate-polyacrylamide gel electrophoresis (SDS-PAGE) and transferred to polyvinylidene difluoride (PVDF) membrane. The membranes were blocked with 5\% skim milk in TBST buffer (20 mM Tris- $\mathrm{HCl}, 150 \mathrm{mM} \mathrm{NaCl}$, and $0.1 \%$ Tween $20, \mathrm{pH} 7.6$ ) for 1 hour at room temperature and then subsequently incubated with primary antibodies including $\mathrm{p}-\mathrm{NF}-\kappa \mathrm{B} / \mathrm{NF}-\kappa \mathrm{B}$ (sc-101752/8242S), p-p38/p38 (sc-17852/sc-535), p-JNK/JNK (sc-6254/sc-7345), and GAPDH (sc-25778) (Santa Cruz Biotechnology, Inc, USA and Cell Signaling Technology, Inc, USA) at $4^{\circ} \mathrm{C}$ overnight. Membranes were then shaken with secondary antibodies for 1 hour at room temperature. Signals were obtained using ECL reagent and detected with UVITEC imaging system equipment. The relative protein expression of $\mathrm{p}-\mathrm{NF}-$ $\kappa \mathrm{B}$ and NF- $\kappa \mathrm{B}, \mathrm{p}-\mathrm{p}-38$ and $\mathrm{p}-38$, and $\mathrm{p}-\mathrm{JNK}$ and JNK (relative to GAPDH) from western blot data was determined using ImageJ.

2.6. Cell Viability Assay. The MTT (3-(4, 5-dimethylthiazol2-yl)-2, 5-diphenyltetrazolium bromide) assay was performed to determine cell viability [3]. HaCaT cells were seeded at a density of $10^{4}$ per $100 \mu$ l culture media in 96-well plates. B. davidii aqueous extract was added at various concentrations from a dilution series from $100 \mu \mathrm{g} / \mathrm{ml}, 50 \mu \mathrm{g} /$ $\mathrm{ml}, 25 \mu \mathrm{g} / \mathrm{ml}, 12.5 \mu \mathrm{g} / \mathrm{ml}, 6.25 \mu \mathrm{g} / \mathrm{ml}, 3.125 \mu \mathrm{g} / \mathrm{ml}$, and $1.5625 \mu \mathrm{g} / \mathrm{ml}$. After incubation for 24 hours, the cells were incubated with a $0.1 \%$ MTT solution in the cell culture medium for 4 hours at $37^{\circ} \mathrm{C}$ in a humidified incubator under $5 \% \mathrm{CO}_{2}$. The MTT solution was discarded, and DMSO was added to solubilize the MTT-formazan crystals produced in the live cells. After incubating in the dark for 2 hours, absorbance was measured at $540 \mathrm{~nm}$ [16]. 
TABLE 1: RT-PCR primer sequences used in this study.

\begin{tabular}{lccc}
\hline Genes & \multicolumn{1}{c}{ Forward } & Reverse & Reference \\
\hline iNOS & TTTCCTTACGAGGCGAAGAAGG & GTTAGGAGGTCAAGTAAAGGGC & {$[11]$} \\
COX-2 & GCTTCCATTGCCAGAGCAGGCA & GAGCTCTGGATCTGGAACACTG & {$[12]$} \\
TLR-2 & TGTCTTGTGACCGCAATGGT & GTTGGACAGGTCAAGGCTTT & {$[3]$} \\
TNF- $\alpha$ & TGAGCACTGAAAGCATGATCC & ATCACTCCAAAGTGCAGCAG & {$[13]$} \\
IL-1 $\beta$ & TCTTTGAAGAAGAGCCCGTCCTC & GGATCCACACTCTCCAGCTGCA & {$[14]$} \\
IL-6 & AGAGTAGTGFAGGAACAAGCC & TACATTTGCCGAAGAGCCCT & {$[15]$} \\
IL-8 & GCAGTTTTGCCAAGGAGTGCT & TTTCTGTGTTGGCGCAGTGTG & {$[3]$} \\
GAPDH & GTGAAGGTVGGAGTVAACG & TGAGGTCAATGAAGGGGTC & {$[16]$} \\
\hline
\end{tabular}

2.7. Statistical Analysis. All data are presented as mean\pm standard deviation (SD) of at least three independent experiments and were analyzed using one-way ANOVA. Student's $t$ test was used when only two groups were compared. Differences were considered statistically significant at $p<0.05, p<0.01$.

\section{Results}

3.1. Screening for Potential Anti-Inflammatory Activity of Aqueous Extract of Plants. The effect of plant aqueous extract on the expression of genes is given in Table 2.

The inflammatory process increases the expression of COX-2 and NO synthase, which produce inflammatory mediators $[12,17]$. In addition, proinflammatory cytokines such as TNF- $\alpha$, IL- $1 \beta$, IL- 6 , and IL- 8 trigger and rapidly amplify the inflammatory response to limit the spreading of the infection $[2,18]$. In this study, 16 plants were extracted from Jeju Island (Korea) that were tested for their antiinflammatory activity against $C$. acnes-induced inflammation. The percentage of gene expression described in Table 2 was calculated based on the ratio of gene expression of $C$. acnes-infected $\mathrm{HaCaT}$ cells treated with aqueous plant extract over one without the plant aqueous extract as an increasing $100 \%$ compared with C. acnes-free HaCaT cells. Reduced values represent the inhibition of the expression of COX-2, NO, and proinflammatory cytokines such as TNF- $\alpha$, IL-1 $\beta$, IL-6, and IL-8. Table 2 shows $A$. japonica A. Gray, $B$. davidii, $I$. verum, and $P$. fibrosa aqueous extract decreased the inflammation process caused by C. acnes. Among them, $B$. davidii aqueous extract showed the strongest inhibition toward the inflammatory process caused by $C$. acnes via reduced iNOS, COX-2, TNF- $\alpha$, IL- $1 \beta$, IL-6, and IL- 8 expression (Table 2).

3.2. Suppression of C. acnes-Induced TLR-2 Expression in $\mathrm{HaCaT}$ Cells by B. davidii Aqueous Extract. C. acnes contribute to the inflammation in acne through activation of toll-like receptors (TLRs), in particular, TLR-2 [2, 19]. In the initial screening, $B$. davidii aqueous extracts displayed the strong decline of the proinflammatory cytokine expressions in C. acnes-treated HaCaT cells (Table 2). The effects of $B$. davidii aqueous extract on expression of the pattern recognition receptor TLR-2 showed $C$. acnes induced the expression of TLR-2 while treated with $50 \mu \mathrm{g} / \mathrm{ml}$ of $B$. davidii aqueous extracts and reduced the TLR-2
mRNA expression by $30 \%$ compared with C. acnes-treated $\mathrm{HaCaT}$ cells without $B$. davidii aqueous extract (Figure 1).

3.3. B. davidii Aqueous Extract Reduced NF-kB Phosphorylation in C. acnes-Treated HaCaT Cells. TLR-2 signaling leads to the activation of the NF- $\kappa$ B pathway, and then $\mathrm{NF}-\kappa \mathrm{B}$ is released and translocates to the nucleus where it regulates expression of an array of genes, which in turn stimulates the release of proinflammatory cytokines such as IL- $1 \beta$, IL- 6 , IL- 8 , and TNF- $\alpha$ [2-4]. The NF- $\kappa$ B pathway was activated by $C$. acnes, indicated by increased expression of toll-like receptor 2 (TLR-2) (Figure 1) compared with $C$. acnes-free. $B$. davidii aqueous extract dose-dependently reduced the amount of p-NF- $\kappa B$ and $\mathrm{NF}-\kappa \mathrm{B}$ protein which could be due to the decrease of the gene expression, as determined using western blotting (Figure 2). These results suggest that the anti-inflammatory activity of $B$. davidii aqueous extract in $\mathrm{HaCaT}$ cells with $C$. acnes-induced inflammation might be due to suppression of the NF- $\kappa \mathrm{B}$ signaling pathway.

3.4. Inhibition of C. acnes-Induced $p 38$ and JNK Expression and Phosphorylation by B. advidii Aqueous Extract. The mitogen-activated protein kinase (MAPK) signaling pathways play an essential role in regulating the production of multiple inflammatory mediators $[2,4,20]$. The effects of $B$. davidii aqueous extract on $C$. acnes-induced MAPK pathway activity were examined using western blotting for $\mathrm{p}$-JNK and p-p38 (Figure 3). C. acnes-induced MAPK activity in HaCaT cells and treatment with $B$. davidii aqueous extract extracts dose-dependently suppressed this MAPK activity. These results indicate that $B$. davidii aqueous extract exerts its anti-inflammatory actions via inhibition of the MAPK signaling pathway.

3.5. B. davidii Aqueous Extract Inhibited the C. acnes-Induced Proinflammatory Cytokine Expression-Induced HaCaT Cells. The primary proinflammatory cytokines are IL- $1 \beta$, IL-6, IL8 , and TNF- $\alpha$ that play important roles in inflammation $[4,18]$. To assess the effects of $B$. davidii aqueous extract on C. acnes-induced proinflammatory cytokine expression, $\mathrm{HaCaT}$ cells were pretreated with indicated concentrations $(12.5,25,50$, and $100 \mu \mathrm{g} / \mathrm{ml})$ of $B$. davidii aqueous extract for 1 hour prior to addition of heat-killed C. acnes (100 MOI) for 24 hours. C. acnes-treated $\mathrm{HaCaT}$ increased the expression 
TABLE 2: Effect of plant aqueous extract on the expression of genes related to inflammatory mediators and proinflammatory cytokines in $C$. acnes-treated $\mathrm{HaCaT}$ cells (unit: \%).

\begin{tabular}{|c|c|c|c|c|c|c|}
\hline Herb & iNOS & COX-2 & TNF- $\alpha$ & IL-1 $\beta$ & IL-6 & IL-8 \\
\hline Akebia quinata & 160 & 218 & 81 & 123 & 131 & 87 \\
\hline Angelica japonica A. Gray & 67 & 26 & 58 & 65 & 38 & 87 \\
\hline Artemisia campestris & 38 & 53 & 44 & 73 & 126 & 105 \\
\hline Buddleja davidii & 41 & 57 & 29 & 32 & 21 & 35 \\
\hline Ceramium kondoi Yendo & 173 & 148 & 115 & 151 & 117 & 95 \\
\hline Chelidonium majus L. var. asiaticum Ohwi & 61 & 54 & 90 & 173 & 112 & 121 \\
\hline Elaeagnus glabra & 50 & 48 & 114 & 211 & 180 & 183 \\
\hline Fallopia japonica (Houtt.) Ronse Decr. & 167 & 238 & 72 & 87 & 109 & 114 \\
\hline Hydrangea macrophylla & 120 & 106 & 18 & 22 & 97 & 25 \\
\hline Hydrangea serrate & 178 & 116 & 86 & 111 & 132 & 161 \\
\hline Hydrangea serrata Seringe & 110 & 90 & 75 & 66 & 83 & 66 \\
\hline Illicium verum & 16 & 73 & 31 & 46 & 61 & 44 \\
\hline Platycladus orientalis & 156 & 114 & 31 & 122 & 15 & 182 \\
\hline Pyllacantha fibrosa & 25 & 77 & 69 & 62 & 36 & 69 \\
\hline Rubus coreanus & 60 & 58 & 95 & 113 & 145 & 179 \\
\hline Sapindus mukorossi & 102 & 99 & 60 & 169 & 127 & 107 \\
\hline
\end{tabular}

Control 1: HaCaT with C. acnes-free and without plants aqueous extract. Control 2: HaCaT with C. acnes and without plant aqueous extract (increasing 100\% compared with control 1).

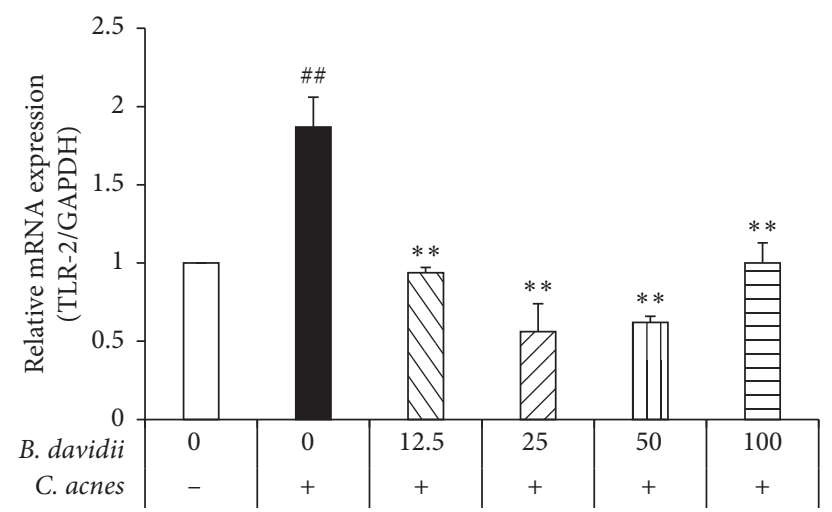

FIgURE 1: B. davidii aqueous extracts inhibited the expression of TLR-2 in C. acnes-treated HaCaT cells. The expression level of TLR-2 mRNA was determined using a RT-PCR. HaCaT cells were seeded in six-well plates for 24 hours and then were pretreated with various concentrations $(12.5,25,50$, and $100 \mu \mathrm{g} / \mathrm{ml})$ of $B$. davidii aqueous extracts for 1 hour and incubated with heat-killed C. acnes (100 MOI) for 24 hours. The values are expressed as the mean \pm SD of three independent experiments, ${ }^{\# \#} p<0.01$ vs $C$. acnes-free and without $B$. davidii aqueous extract, ${ }^{*} p<0.05,{ }^{*} p<0.01$ vs $C$. acnes and without plants aqueous extract.

of IL- $1 \beta$, IL-6, IL- 8 , and TNF- $\alpha$. However, the expression of IL-1 $\beta$, IL-6, IL-8, and TNF- $\alpha$ was dose-dependently declined in $C$. acnes-treated cells after treatment with $B$. davidii aqueous extract (Figure 4). IL-1 $\beta$, IL-6, IL-8, and TNF- $\alpha$ expression was decreased by approximately $32 \%, 21 \%, 35 \%$, and $29 \%$ at $50 \mu \mathrm{g} / \mathrm{ml}$, respectively, compared with untreated cells. Hence, $50 \mu \mathrm{g} / \mathrm{ml}$ of $B$. davidii aqueous extract is the highest concentration of the extract shows the sharpest effect. These results indicate that $B$. davidii aqueous extract could have anti-inflammatory effects through inhibiting proinflammatory cytokine expression.

3.6. Cytotoxicity of B. davidii Aqueous Extract in HaCaT C. To determine the cytotoxic effects of $B$. davidii aqueous extracts on $\mathrm{HaCaT}$ cells, the MTT assay was used. The cells were treated with $B$. davidii aqueous extracts for 24 hours at a concentration ranging from $0.39 \mu \mathrm{g} / \mathrm{ml}$ to $100 \mu \mathrm{g} / \mathrm{ml}$, and the $B$. davidii aqueous extracts had no significant cytotoxic effects on HaCaT cells (Figure 5).

\section{Discussion}

Inflammation has been suggested as a key factor involved in the development and aggravation of acne vulgaris, although the exact pathogenesis of acne vulgaris has not been elucidated. It has been verified that $C$. acnes play a major role in the development of inflammatory acne lesions [1, 4]. C. acnes promote the activation of TLR-2 in keratinocytes, aggravating the inflammation reactions $[2,4]$. In fact, the $C$. acnes infection begins to activate monocyte TLR-2, a major signaling pathway induced by activation of NF- $\kappa$ B or MAPKs signaling pathway, which in turn gives rise to expression of cytokines, chemokines, adhesion molecules, and 


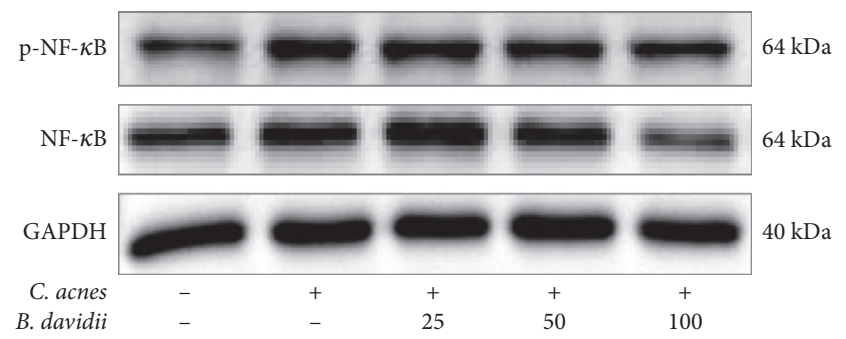

(a)

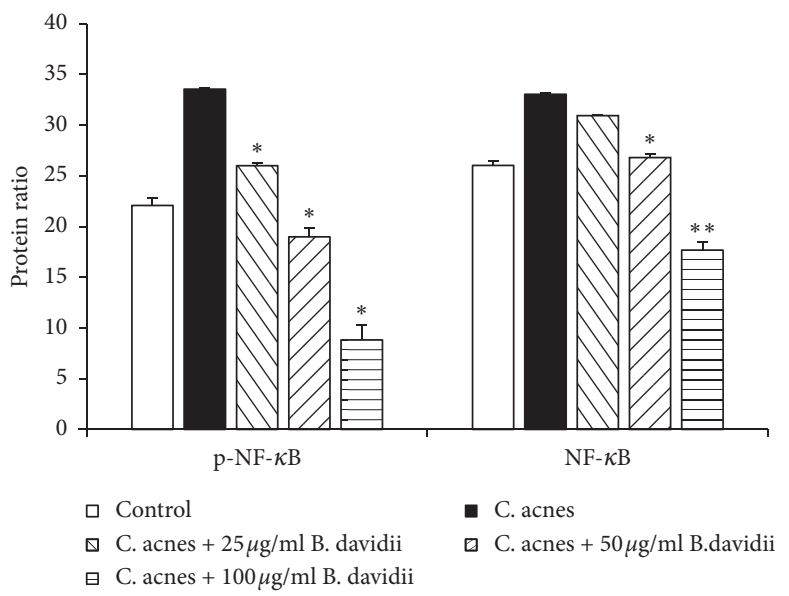

(b)

Figure 2: Reduction of the protein ratio of NF- $\kappa \mathrm{B}$ in $C$. acnes-treated HaCaT cells by $B$. davidii aqueous extracts. HaCaT cells were pretreated with the indicated concentration of $B$. davidii aqueous extracts for 1 hour before treatment with heat-killed C. acnes (100 MOI) for 24 hours. Western blot analysis was carried out to evaluate the level of phosphorylated NF- $\kappa$ B or NF- $\kappa$ B. (a) B. davidii aqueous extracts reduced the phosphorylation of NF- $\kappa \mathrm{B}$ and C. acnes-induced inflammation. (b) Relative protein expression of p-NF- $\kappa \mathrm{B}$ and NF- $\kappa \mathrm{B}$ (relative to GAPDH) was determined using densitometry. Control was C. acnes-free without B. davidii aqueous extract. All data were expressed as mean \pm SD. ${ }^{*} p<0.05$ compared with $C$. acnes treated cells only.

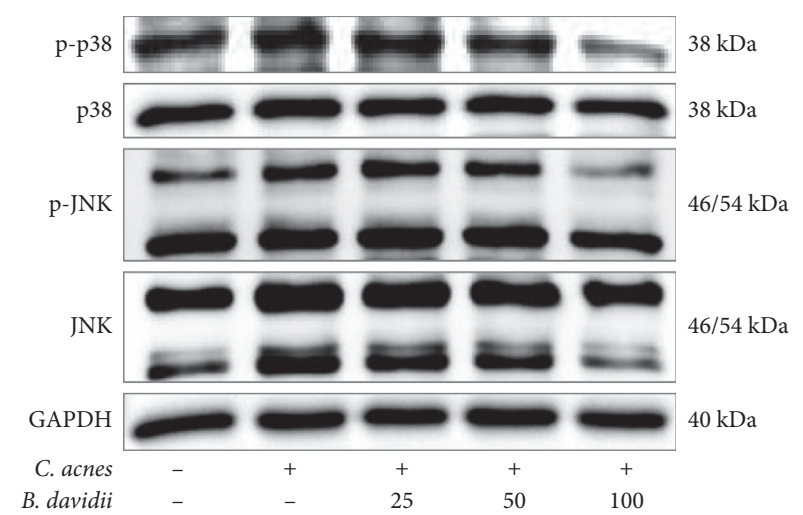

(a)

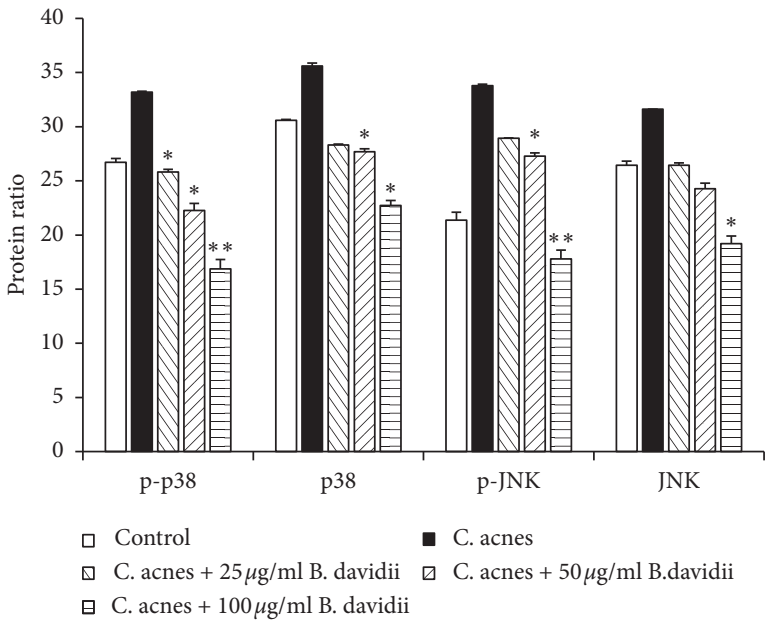

(b)

FIgURE 3: Inhibition of $B$. davidii aqueous extract on MAPKs expression in $C$. acnes-treated HaCaT cells. Western blot analysis was used to check the amount of protein of p-p38, p38, p-JNK, and JNK. HaCaT cells were pretreated with the indicated concentration of $B$. davidii aqueous extracts for 1 hour before treatment with heat-killed C. acnes (100 MOI) for 24 hours. (a) Total protein expression and phosphorylation of p38 and JNK were reduced by treatment with $B$. davidii aqueous extracts. (b) Relative protein expression of p-p38, p38, pJNK, and JNK (relative to GAPDH) was determined using ImageJ. All data were expressed as mean \pm SD. ${ }^{*} p<0.05,{ }^{* *} p<0.01$ compared with C. acnes-treated cells only.

granulopoiesis factors $[4,18,20]$. On the basis of this information, $B$. davidii aqueous extract effectively prevents the inflammation caused by $C$. acnes through inhibition of the TLR-2 to NF- $\kappa$ B signaling cascade in keratinocytes (Figures 1 and 2).

Recently, it has been studied that the MAPK signaling pathway plays a critical role in regulation of the inflammatory response and coordination of the induction of many genes encoding inflammatory mediators
$[13,19,20]$. B. davidii aqueous extract suppressed mitogen-activated protein kinase (MAPK) by inhibiting p-p38 and p-JNK in HaCaT cells treated with C. acnes (Figure 3). Activation of the TLR-2-mediated downstream MAPK signaling pathway is responsible for production of inflammatory cytokines. MAPK activation has been found in clinical acne lesions, indicating that inhibition of MAPK signaling is important for the pathogenesis of acne vulgaris in general $[4,20]$. Use of MAPK inhibitors is 


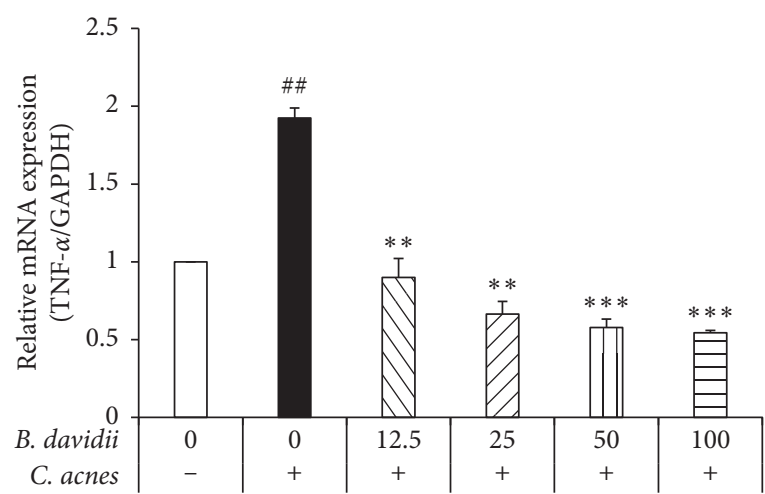

(a)

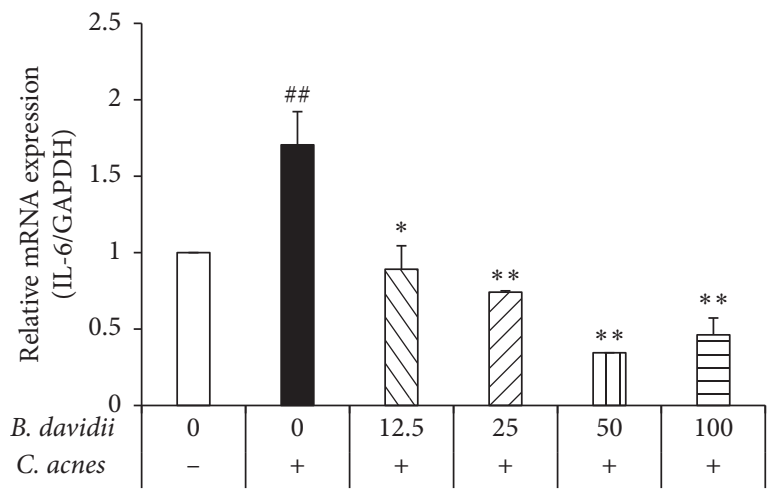

(c)

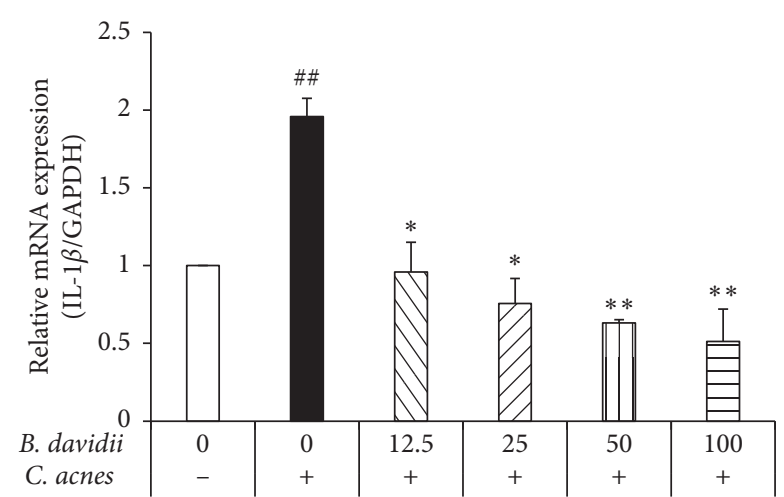

(b)

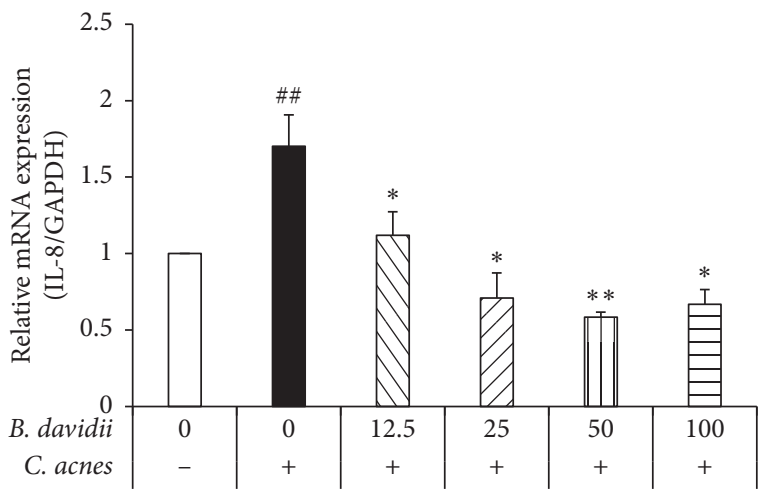

(d)

Figure 4: $B$. davidii aqueous extract reduced the level of the proinflammatory cytokines expression in $C$. acnes-treated HaCaT cells. $\mathrm{HaCaT}$ cells were pretreated with $B$. davidii aqueous extract $(12.5,25,50$, and $100 \mu \mathrm{g} / \mathrm{ml}) 1$ hour before incubation with $C$. acnes (100 MOI) for 24 hours. The expression of TNF- $\alpha$, IL-1 $\beta$, IL-6, and IL-8 mRNA was then measured using RT-PCR. Experiments were performed in triplicate using independent samples, and the data are presented as the mean $\pm \mathrm{SD},{ }^{\# \#} p<0.01$ vs $C$. acnes-free and without $B$. davidii aqueous extract, ${ }^{*} p<0.05,{ }^{* *} p<0.01,{ }^{* * *} p<0.001$ vs $C$. acnes and without plant aqueous extract.

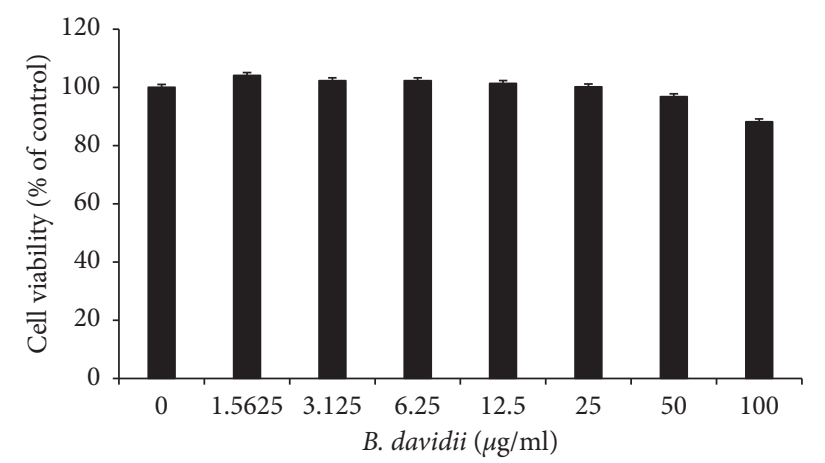

Figure 5: Effects of $B$. davidii aqueous extracts on $\mathrm{HaCaT}$ cell viability. HaCaT cells $\left(10^{4}\right.$ per well) were incubated with $B$. davidii aqueous extracts in 96-well plates for 24 hours, and cell viability was evaluated using the MTT assay. Data represent three independent experiments.

emerging as an attractive alternative to anti-inflammatory drugs because they decrease synthesis of proinflammatory cytokines [20]. The results of this study suggest that $B$. davidii aqueous extract modulates the expression of proinflammatory cytokines (TNF- $\alpha$, IL- $1 \beta$, IL-6, and IL-8) in the inflammation process through the inhibition of the NF- $\kappa \mathrm{B}$ and MAPK kinase pathways (Figures 4 and 6). Hence, $B$. davidii aqueous extract might be a potential
MAPK inhibitor to treat the inflammation caused by bacterial infections.

Previously, $R$. coreanus, S. mukorossi, and P. orientalis aqueous extract had been reported to be anti-inflammatory [21-23]; however, in this study, R. coreanus, S. mukorossi, and $P$. orientalis aqueous extract did not exhibit their antiinflammatory activity (Table 2). In addition, A. japonica A. Gray, B. davidii, I. verum, and P. fibrosa aqueous extract 


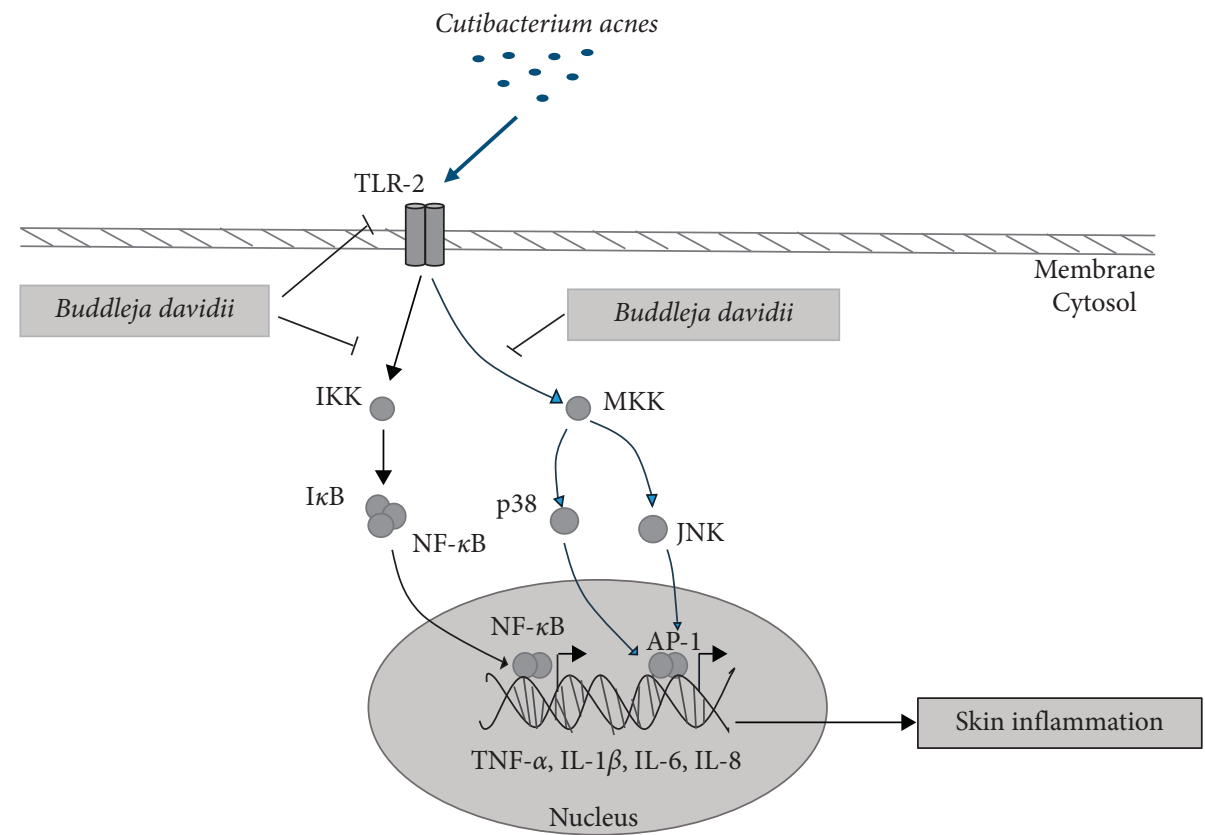

Figure 6: A proposed mechanism of $B$. davidii against $C$. acnes-induced skin inflammation. B. davidii inhibits the inflammation by suppressing the NF- $\kappa \mathrm{B}$ and MAPK signaling pathways, which are activated by TLR-2, leading to the reduced expression of the proinflammatory cytokines TNF- $\alpha$, IL- $1 \beta$, IL-6, and IL-8.

showed an inhibition potential to the inflammation caused the $C$. acnes (Table 2). Here, B. davidii aqueous extract showed a stronger inhibition toward the inflammatory process caused by $C$. acnes than $I$. verum which was reported in the previous studies [15], A. japonica A. Gray, and $P$. fibrosa (Table 2).

B. davidii is a well-known, traditional, herbal medicine and a promising candidate for the treatment of acne and other inflammatory diseases $[6,7]$. A better understanding of the mechanism of $B$. davidii's anti-inflammatory activity would not only offer insight into inflammation homeostasis but also provide opportunities for the discovery of new antiinflammatory compounds with fewer side effects in the future.

In conclusion, this study provides the first evidence that $B$. davidii has the anti-inflammation property through inhibiting two pathways that are NF- $\kappa \mathrm{B}$ and MAPK kinase, leading to the reduced proinflammatory cytokine expression. These findings suggest that $B$. davidii is a potential effective therapeutic agent for the treatment of acne vulgaris. However, further studies are needed for a full understanding of its mechanism of action as well as identification of the active components of $B$. davidii in anti-inflammatory activities such as luteolin, quercentin, hesperetin, and colchicine $[6,24]$. In addition, evaluation of the in vivo effects of $B$. davidii is required for use in clinical applications.

\section{Data Availability}

No data were used to support this study.

\section{Conflicts of Interest}

The authors declare that they have no conflicts of interest.

\section{Acknowledgments}

This research was funded by the GRRC Program of Gyeonggi Province (GRRC-Kyung Hee 2018 (B04)), Republic of Korea.

\section{References}

[1] S. Mollerup, J. Friis-Nielsen, L. Vinner et al., "Propionibacterium acnes: disease-causing agent or common contaminant? Detection in diverse patient samples by nextgeneration sequencing," Journal of Clinical Microbiology, vol. 54, no. 4, 2016.

[2] C. T. Nguyen, S. K. Sah, C. C. Zouboulis, and T.-Y. Kim, "Inhibitory effects of superoxide dismutase 3 on Propionibacterium acnes-induced skin inflammation," Scientific Reports, vol. 8, no. 1, p. 4024, 2018.

[3] S. Ryu, H. M. Han, P. I. Song, C. A. Armstrong, and Y. Park, "Suppression of Propionibacterium acnes infection and the associated inflammatory response by the antimicrobial peptide P5 in mice," PLoS One, vol. 10, no. 7, Article ID e0132619, 2015.

[4] M. L. Schmitz and S. Bacher, "Novel molecular targets in the search for anti-inflammatory agents," Phytochemistry Reviews, vol. 4, no. 1, pp. 19-25, 2005.

[5] WHO, "Chapter 5: traditional and complementary medicine policy," MDS-3: Managing Access to Medicines and Health Technologies, Management Sciences Health, 2012.

[6] J. Wu, W. Yi, L. Jin, D. Hu, and B. Song, "Antiproliferative and cell apoptosis-inducing activities of compounds from Buddleja davidii in Mgc-803 cells," Cell Division, vol. 7, no. 1, p. 20, 2012.

[7] P. J. Houghton and A. Y. Mensah, "Chapter 13: biologically active compounds from Buddleja species," Phytochemicals in Human Health Protection, Nutrition, and Plant Defense, London, UK, 1999. 
[8] S. N. H. Mohammad Azmin, Z. Abdul Manan, S. R. Wan Alwi, L. S. Chua, A. A. Mustaffa, and N. A. Yunus, "Herbal processing and extraction technologies," Separation \& Purification Reviews, vol. 45, no. 4, pp. 305-320, 2016.

[9] R. Nagappan, "Evaluation of aqueous and ethanol extract of bioactive medicinal plant, Cassia didymobotrya (Fresenius) Irwin \& Barneby against immature stages of filarial vector, Culex quinquefasciatus Say (Diptera: Culicidae)," Asian Pacific Journal of Tropical Biomedicine, vol. 2, no. 9, pp. 707-711, 2012.

[10] J. Kim, Y.-K. Shin, and K.-Y. Kim, "Promotion of keratinocyte proliferation by tracheloside through ERK1/2 stimulation," Evidence-based Complementary and Alternative Medicine, vol. 2018, Article ID 4580627, 5 pages, 2018.

[11] V. Fetz, C. Bier, N. Habtemichael et al., "Inducible NO synthase confers chemoresistance in head and neck cancer by modulating survivin," International Journal of Cancer, vol. 124, no. 9, pp. 2033-2041, 2009.

[12] H. Zhou, V. N. Ivanov, J. Gillespie et al., "Mechanism of radiation-induced bystander effect: role of the cyclooxygenase-2 signaling pathway," Proceedings of the National Academy of Sciences, vol. 102, no. 41, pp. 14641-14646, 2005.

[13] H. I. Kim, S. H. Hong, J. M. Ku et al., "Tonggyu-tang, a traditional Korean medicine, suppresses pro-inflammatory cytokine production through inhibition of MAPK and NF- $\kappa \mathrm{B}$ activation in human mast cells and keratinocytes," $B M C$ Complementary and Alternative Medicine, vol. 17, no. 1, 2017.

[14] H. J. Lim, Y. D. Jeon, S. H. Kang et al., "Inhibitory effects of Euphorbia supine on Propionibacterium acnes-induced skin inflammation in vitro and in vivo," BMC Complementary and Alternative Medicine, vol. 18, no. 263, 2018.

[15] Y.-Y. Sung, Y. S. Kim, and H. K. Kim, "Illicium verum extract inhibits TNF- $\alpha$ - and IFN- $\gamma$-induced expression of chemokines and cytokines in human keratinocytes," Journal of Ethnopharmacology, vol. 144, no. 1, pp. 182-189, 2012.

[16] P. A. Grange, C. Chéreau, J. Raingeaud et al., "Production of superoxide anions by keratinocytes initiates $P$. acnes-induced inflammation of the skin," PLoS Pathogens, vol. 5, no. 7, Article ID e1000527, 2009.

[17] O. O. Oluwafemi, "Medicinal plants with anti-inflammatory activities from selected countries and regions of Africa," Journal of Inflammation Research, vol. 11, pp. 307-317, 2018.

[18] J.-M. Zhang and J. An, "Cytokines, inflammation, and pain," International Anesthesiology Clinics, vol. 45, no. 2, pp. 27-37, 2007.

[19] Y.-C. Huang, C.-H. Yang, T.-T. Li, C. C. Zouboulis, and H.-C. Hsu, "Cell-free extracts of Propionibacterium acnes stimulate cytokine production through activation of p38 MAPK and toll-like receptor in SZ95 sebocytes," Life Sciences, vol. 139, pp. 123-131, 2015.

[20] B. Kaminska, "MAPK signaling pathways as molecular targets for anti-inflammatory therapy-from molecular mechanisms to therapeutic benefits," Biochim Biophys Acta, vol. 1754, no. 1-2, pp. 253-262, 2005.

[21] J. E. Lee, S.-M. Cho, E. Park et al., "Anti-inflammatory effects of Rubus coreanus Miquel through inhibition of NF- $\kappa \mathrm{B}$ and MAP Kinase," Nutrition Research and Practice, vol. 8, no. 5, pp. 501-508, 2014.

[22] M. Shah, Z. Parveen, and M. R. Khan, "Evaluation of antioxidant, anti-inflammatory, analgesic and antipyretic activities of the stem bark of Sapindus mukorossi," BMC Complementary and Alternative Medicine, vol. 17, no. 1, p. 526, 2017.
[23] S.-Y. Fan, H.-W. Zeng, Y.-H. Pei et al., "The anti-inflammatory activities of an extract and compounds isolated from Platycladus orientalis (Linnaeus) Franco in vitro and ex vivo," Journal of Ethnopharmacology, vol. 141, no. 2, pp. 647-652, 2012.

[24] S. Khan, H. Ullah, and L. Zhang, "Bioactive constituents form Buddleja species," Pakistan Journal of Pharmaceutical Science, vol. 32, no. 2, pp. 721-741, 2019. 